\title{
The risk for subsequent coronary interventions in a local Polish population
}

\author{
Jaroslaw Hiczkiewicz ${ }^{1,2}$, Paweł Burchardt ${ }^{3,4}$, Konrad Pieszko ${ }^{1,2}$, Jan Budzianowski ${ }^{1,2}$, Dariusz Hiczkiewicz ${ }^{1,2}$, \\ Bogdan Musielak ${ }^{1,2}$, Anna Winnicka-Zielinska ${ }^{1,2}$, Daria Adamczak ${ }^{5}$, Wojciech Faron ${ }^{1}$, Janusz Rzeźniczak ${ }^{4}$
}

${ }^{1}$ Department of Cardiology, Multidisciplinary District Hospital, Nowa Sol, Poland

${ }^{2}$ Faculty of Medicine and Health Sciences, University of Zielona Gora, Zielona Gora, Poland

${ }^{3}$ Department of Hypertension, Angiology, and Internal Medicine, Poznan University of Medical Sciences, Poznan, Poland

${ }^{4}$ Department of Cardiology, J. Strus Hospital, Poznan, Poland

$\left.{ }^{5}\right|^{\text {st }}$ Department of Cardiology, Poznan University of Medical Sciences, Poznan, Poland

Adv Interv Cardiol 2020; 16, 4 (62): 429-435

DOI: https://doi.org/10.5114/aic.2020.101768

\begin{abstract}
A bstract
Introduction: Paradoxically, the literature lacks an assessment of the impact of various factors on subsequent coronary interventions in patients with coronary artery disease (CAD).

Aim: To assess the impact of various factors on subsequent percutaneous transluminal coronary angioplasty (PTCA), as well as to characterize the clinical profile of people undergoing repeated diagnostic coronary angiography without significant coronary artery changes.

Material and methods: We investigated retrospective data from 4041 subjects according to the clinical factors which may affect the occurrence of unplanned future PTCA.

Results: The strongest risk factors for subsequent PTCA were significant stenosis of left descending artery $(\mathrm{OR}=2.17,95 \% \mathrm{Cl}$ : 1.09-4.32) during baseline coronary angiography, the atherosclerotic burden (number of critically narrowed vessels) (OR for narrowing lesions in 3 epicardial arteries $12.13,95 \% \mathrm{Cl}$ : 5.40-27.27), and restenosis in a previously implanted stent $(\mathrm{OR}=4.34,95 \% \mathrm{Cl}$ : 1.96-9.62). A strong positive relationship between total mortality and the number of critically narrowed coronary arteries (during baseline hospitalization) was observed. Patients without significant coronary artery stenosis in two diagnostic angiographies (control group) differed from subjects with hemodynamic relevant CAD in: higher creatinine levels, more frequent presence of chronic obstructive pulmonary disease and more frequent symptoms of intermittent claudication.

Conclusions: The results of the study are in accord with real clinical practice. The arteriosclerotic burden is a major cause of recurrent PTCA, but an important clinical issue is the qualification for recurrent coronary-angiography in those patients whose previous coronary angiography did not show significant stenosis, because other clinical causes may explain their symptoms.
\end{abstract}

Key words: subsequent percutaneous transluminal coronary angioplasty, coronary artery disease, ischemic heart disease.

Su m mary

The available literature lacks an assessment of the impact of various factors on subsequent coronary interventions in patients with coronary artery disease (CAD). We found that the strongest risk factors for subsequent percutaneous transluminal coronary angioplasty in our analysis were significant narrowed left descending artery, restenosis in a previously implanted stent, and atherosclerotic burden. Only a few reports are available concerning characterizing the clinical profile of patients undergoing diagnostic coronary angiographies on several occasions with no significant changes in the lumen of the coronary arteries. Patients with symptoms suggestive of angina without significant coronary artery stenosis in angiography differed from patients with CAD in our study by higher creatinine levels, more frequent presence of chronic obstructive pulmonary disease and more frequent symptoms of intermittent claudication.

\section{Corresponding author:}

Prof. Paweł Burchardt, Department of Hypertension, Angiology, and Internal Medicine, Poznan University of Medical Sciences; Department of Cardiology, J. Strus Hospital, Poznan, Poland, phone: +48 606611 011, e-mail: pab2@tlen.pl

Received: 5.08.2020, accepted: 1.10.2020. 


\section{Introduction}

Cardiovascular disease (CVD) is the leading cause of mortality worldwide (about $50 \%$ of all deaths) [1]. In 2017, the number of people who died due to CVD in Poland was 150,000 [2]. It is estimated that $98 \%$ of cases of ischemic heart disease (IHD), which accounts for approximately $50 \%$ of cardiovascular deaths, are caused by atherosclerosis [3]. In 2018, 182226 diagnostic coronary angiographies and 104283 coronary angioplasties (as a coronary artery disease treatment) were performed in Poland [4].

It is estimated that up to $30 \%$ of patients who have undergone coronary angioplasty continue to report chest pain despite optimal pharmacotherapy [5-7]. Recurrent angina may be caused by the natural progression of atherosclerosis, complications from previous procedures, improper stent implantation technique, and the commonly known phenomenon of restenosis. The symptoms often lead to further cardiovascular interventions [5-7], sometimes prematurely.

\section{Aim}

The available literature lacks an assessment of the impact of various factors on subsequent coronary interventions in patients with coronary artery disease (CAD), which is the first goal of this study.

On the other hand, there are few reports characterizing the clinical profile of patients undergoing diagnostic coronary angiographies on several occasions with no significant changes in the lumen of the coronary arteries. This study's second goal is to identify attributes/traits that are typical for this group of people in a local Polish population.

\section{Material and methods}

\section{Methodology}

The study included 4041 patients with established or suspected CAD who had at least two invasive procedures in the period between January $14^{\text {th }}, 2009$ and May $15^{\text {th }}$, 2015 at the Cardiology Department in Nowa Sol. Index hospitalization took place during the second intervention, whenever it was just coronary angiography or coronary angioplasty (PTCA). Patients were divided into groups based on the history of cardiovascular interventions.

We defined the control group (CG) as patients who had at least two coronary angiographies performed and each time no significant lesions were found ( $n=640$ ).

The remaining 3401 patients underwent at least two coronary angioplasties. The index hospitalization took place during the second PTCA. During that all of significantly narrowed coronary arteries were supplied and if needed also additional PTCA was performed during the follow-up period. In this group we distinguished a subpopulation of 2445 subjects who underwent 2 angioplasties, 751 people who had three angioplasties, 155 people had four angioplasties and 50 people had five or more angioplasties. Patients who had undergone only one PTCA were excluded.

The mean weighted (according to number of participants in groups) period between index PTCA and subsequent ones: first coronary angioplasty was 154 days, second coronary angioplasty 181.5 days, third PTCA was 193 days.

Data obtained from patients undergoing coronary index angioplasty were analyzed for:

- specific anatomical and morphological features present in coronary arteries,

- type of techniques used during PTCA procedure,

- specific electrocardiographic, echocardiographic and laboratory features present during index hospitalization determining the necessity of performing further PTCA in the studied population, as well as differentiating people with and without a history of coronary intervention.

Additionally, the studied group of 3401 patients who had undergone PTCA during index hospitalization was related to the presence of lesions in one, two, or three coronary arteries.

The exclusion criteria from the study were as follows: - history of coronary artery bypass graft (CABG) or qualification for this procedure during the observation period, - significant stenosis of the left main,

- coexisting heart defects,

- concomitant severe NYHA III/IV heart failure.

The study flow chart is presented below.

\section{Laboratory assessment}

The blood was collected during every intervention, but for analysis laboratory data obtained during the second angiography were used. In the analysis the peripheral blood count was determined with a CELL-DYN Ruby analyzer (Abbott Diagnostics, USA). TnT was measured with a Cobas 6000 device (Roche Diagnostics $\mathrm{GmbH}$ ) with a cut-off value of $14 \mathrm{pg} / \mathrm{l}$. Creatinine, total cholesterol, triglycerides and high-density lipoproteins were analyzed by means of a photometric test (Roche Diagnostics $\mathrm{GmbH}$ ).

\section{Statistical analysis}

The analyzed data came from an interval and nominal scale. Interval data such as age or blood biochemical results were presented as mean and standard deviation $(x \pm \mathrm{SD})$. Comparisons were made between more than two groups; one-way analysis of variance (ANOVA) was used together with Tukey's post-hoc test or the Shapiro-Wilk test and Levene's test; the Kruskal-Wallis test with post-hoc Dunn's test was used alternatively. In the following, one-dimensional logistic regression models were used, followed by multi-dimensional logistic regression models. The results obtained were presented as the odds ratio (OR) with a 95\% confidence interval. Survival 
analysis results are presented graphically as Kaplan-Meier curves. Statistical analysis was performed using the STATA 15.1 program (StataCorp LLC)/resource of Poznan University of Medical Science/). All tests were analyzed at the significance level $\alpha=0.05$.

All patients with established CAD were treated pharmacologically and invasively, according to either the current guidelines for the management of stable coronary artery disease or the revascularization guidelines of the European Society of Cardiology. However, many of the therapy goals were not achieved. Recommended pharmacotherapy during index hospitalization, at discharge was as follows. Salicylates, statins, angiotensin converting enzyme, beta blockers, clopidogrel, hypoglycemic agents, ticagrelor, calcium channel blockers, diuretics/nitrates and prasugrel were prescribed respectively in $98 \%, 97.4 \%, 89.2 \%, 81.1 \%$, $79.8 \%, 17.3 \%, 16.3 \%, 5.2 \%, 2.5 \%, 1.5 \%$ of cases.

The study was retrospective, carried out in accordance with the principles of the Helsinki Declaration, and the protocol was accepted by the bioethics committee.

\section{Results}

The study included 4041 patients with established or suspected CAD. They were divided into two groups. The CAD group contained 3401 patients (between 24 and 96 years of age) who underwent at least angioplasty (of all significantly narrowed coronary arteries), twice. The reasons for index hospitalization were as follows: acute coronary syndrome (ACS) ST elevation myocardial infarction in $12.5 \%$, non-ST elevation myocardial infarction in $4.9 \%$, unstable angina in $19.4 \%$, and stable angina in $63.2 \%$ of cases.

The control group included 640 subjects (between 44 and 97 years of age) with suspicion of stable angina, who had at least two coronary angiographies performed during the follow-up period and each time no significant lesions were found. The characteristics of studied groups are shown in Table I.

Patient characteristics due to the number of percutaneous coronary angioplasties

During index angioplasty restenosis of previously implanted stent(s) (history of PTCA) was observed in $9.8 \%$ of cases. Predilatation was performed in $33.1 \%$. The direct stenting technique was used in 60\%. During angioplasty drug-eluting stent (DES)/bare metal stents (BMS) or plain old balloon angioplasty (POBA) was applied respectively in $53.8 \%$ vs. $47.7 \%$ vs. $6.9 \%$. Major procedural complications (dissection or perforation) following the PTCA were observed in $2.2 \%$. Clinical complications such as myocardial infarction type 4 a or ventricular fibrillation were observed in $2.23 \%$ of CAD subjects (Table II).

Patient characteristics including differences between significant lesions in one coronary artery vs. multivessel disease, noted during index hospitalization

Patients with significant stenosis in one or two coronary arteries were significantly younger than those with three-vessel disease and co-branches (age: $64.3 \pm 9.7 \mathrm{a}$, $64.9 \pm 9.5 a$ vs. $69.4 \pm 9.9 b, p=0.0004)$ and had lower creatine levels (creatine $(\mathrm{mg} / \mathrm{dl}) 1.0 \pm 0.4 \mathrm{a}, 1.0 \pm 0.5 \mathrm{a}$ vs. 1.24 $\pm 0.58, p=0.0213$ ) ( $a, b-$ groups followed by the same letter do not differ statistically significantly at the significance level $\alpha=0.05$ ).

The impact of factors analyzed during index angioplasty on subsequent coronary interventions (multifactorial regression)

This model compared the data obtained during first index hospitalization in which angioplasty of all signifi-

Table I. Characteristics of studied population

\begin{tabular}{|c|c|c|c|}
\hline Parameter & $\begin{array}{l}\text { Coronary artery disease group } \\
\qquad(N=3401)\end{array}$ & $\begin{array}{l}\text { Control group/without CAD/ } \\
\qquad(N=640)\end{array}$ & $P$-value \\
\hline Age [years] & $64.8 \pm 9.8$ & $66.6 \pm 8.9$ & 0.0005 \\
\hline Male sex (\%) & 68 & 61.7 & 0.008 \\
\hline Hypertension (HA) (\%) & 92.7 & 82 & $<0.001$ \\
\hline Diabetes (DM) (\%) & 24 & 21 & $>0.05$ \\
\hline Heart insufficiency by NYHA I/II (\%) & 10 & 15 & 0.001 \\
\hline Chronic kidney disease (CKD) (\%) & 3 & 15 & $<0.0001$ \\
\hline Chronic obstructive pulmonary disease (COPD) (\%) & 4 & 12 & $<0.0001$ \\
\hline Intermittent claudication & 6 & 12.8 & $<0.0001$ \\
\hline Dyslipidemia (\%) & 26 & 40 & $<0.0001$ \\
\hline Stroke (\%) & 4.7 & 2.3 & 0.02 \\
\hline Smoking (\%) & 11.6 & 15 & 0.008 \\
\hline
\end{tabular}


Table II. Characteristics according to number of PTCA performed

\begin{tabular}{|c|c|c|c|c|c|}
\hline \multirow[t]{2}{*}{ Parameter } & \multicolumn{4}{|c|}{ Number of PTCA } & \multirow[t]{2}{*}{$P$-value } \\
\hline & $\begin{array}{l}\text { Index hospitalization } \\
\text { (second PTCA) } \\
(N=3401)\end{array}$ & $\begin{array}{l}\text { First angioplasty after } \\
\text { index one (third PTCA) } \\
\qquad(N=751)\end{array}$ & $\begin{array}{l}\text { Second angioplasty } \\
\text { after index one } \\
\text { (fourth PTCA) } \\
(N=155)\end{array}$ & $\begin{array}{l}\text { Third and more angio- } \\
\text { plasties after index one } \\
\text { (fifth or more PTCA) } \\
(N=50)\end{array}$ & \\
\hline Age [years] & $65 \pm 9.9^{a}$ & $64.08 \pm 9.04^{\mathrm{a}, \mathrm{b}}$ & $63.3 \pm 9.4^{\mathrm{b}, \mathrm{c}}$ & $62.3 \pm 9.7 c$ & 0.0521 \\
\hline Male sex (\%) & 68 & 71.4 & 73 & 70 & $>0.05$ \\
\hline Stroke (\%) & 4.7 & 4.3 & 5.2 & 0.0 & $>0.05$ \\
\hline $\mathrm{TC}[\mathrm{mg} / \mathrm{dl}]$ & $188.5 \pm 43.3^{\mathrm{a}}$ & $190.4 \pm 42.5^{\mathrm{a}}$ & $200.4 \pm 12.6^{a, b}$ & $211.4 \pm 12.3^{b}$ & $<0.0001$ \\
\hline LDL [mg/dl] & $115.4 \pm 42.5^{a}$ & $118.5 \pm 41.5^{\mathrm{a}}$ & $125.4 \pm 41.8^{a, b}$ & $142.1 \pm 43.4^{b}$ & 0.0006 \\
\hline $\mathrm{HDL}[\mathrm{mg} / \mathrm{dl}]$ & $43.8 \pm 13.2^{b}$ & $42.0 \pm 11.4^{a, b}$ & $41.2 \pm 11.8^{a, b}$ & $38.4 \pm 13.5^{\mathrm{a}}$ & 0.03 \\
\hline $\mathrm{TG}[\mathrm{mg} / \mathrm{dl}]$ & $139.8 \pm 67.3^{\mathrm{a}}$ & $150.4 \pm 58.5^{\mathrm{a}, \mathrm{b}}$ & $170.0 \pm 63.2^{b, c}$ & $196.8 \pm 76.1^{c}$ & $<0.0001$ \\
\hline Creatine [mg/dl] & $1.5 \pm 0.5$ & $1.03 \pm 0.48$ & $1.09 \pm 0.5$ & $441.1 \pm 0.6$ & $>0.05$ \\
\hline EF (\%) & $43.2 \pm 12.8^{a}$ & $45.6 \pm 11.4^{\mathrm{a}, \mathrm{b}}$ & $47.8 \pm 10.6^{b}$ & $48.9 \pm 14.4^{b}$ & 0.002 \\
\hline
\end{tabular}

a,b,c,Groups followed by the same letter do not differ statistically significantly at the significance level $\alpha=0.05 . T C-$ total cholesterol, LDL - low-density lipoprotein, $H D L$ - high-density lipoprotein, TG - triglycerides, EF-ejection fraction.

cantly narrowed coronary arteries was performed with data obtained from patients during subsequent coronary interventions.

The strongest risk factors for subsequent PTCA (multivariate analysis) in patients were: restenosis in a previously implanted stent $(\mathrm{OR}=4.34,95 \% \mathrm{Cl}$ : 1.96-9.62), significant stenosis of left descending artery $(\mathrm{OR}=2.17$, 95\% Cl: 1.09-4.32) during baseline coronary angiography, and the atherosclerotic burden (OR for narrowing lesions in 3 epicardial arteries $12.13,95 \% \mathrm{Cl}: 5.40-27.27)$, in 3 arteries with co-branches ( $\mathrm{OR}=3.45,95 \% \mathrm{Cl}: 0.73-16.27)$, in 2 epicardial arteries (OR $=5.27,95 \% \mathrm{Cl}: 2.51-11.09)$.

The results of one-dimensional analysis showed that additional predilatation during index angioplasty was a risk factor for subsequent PTCA in future $(O R=1.38$, 95\% Cl: 1.17-1.63 for another angioplasty after the index one, $\mathrm{OR}=1.75,95 \% \mathrm{Cl}: 1.26-2.43$ for $2^{\text {nd }}$ angioplasty, and $\mathrm{OR}=2.37,95 \% \mathrm{Cl}: 1.35-4.16$ for further interventions). The types of implanted stents (BMS vs. DES), direct stenting and postdilatation technique had no influence on PTCA in the future.

\section{Assessment of individual parameters between the group of people who were diagnosed with one-vessel disease vs. multivessel disease during index hospitalization}

This model compared the data obtained during the first index hospitalization from subjects with angioplasty of one narrowing artery vs. multivessel disease. One dimensional analysis showed that patients with multivessel disease more often suffered from diabetes, $O R=1.26$, $95 \% \mathrm{Cl}: 1.07-1.47$, heart failure $\mathrm{OR}=1.51,95 \% \mathrm{Cl}: 1.23-$ 1.84 , chronic kidney disease $\mathrm{OR}=1.46,95 \% \mathrm{Cl}: 1.09$
1.94, dyslipidemia OR $=1.26,95 \% \mathrm{Cl}: 1.06-1.49$. During index hospitalization they more often developed: non-ST elevation myocardial infarction, $\mathrm{OR}=3.05$, 95\% Cl: 2.24.22, and restenosis, $\mathrm{OR}=1.61,95 \% \mathrm{Cl}: 2.65-9.79$. Their significant lesions were likely localized on bifurcations, $\mathrm{OR}=4.59,95 \% \mathrm{Cl}: 2.67-7.89$, so they more often need pre- and postdilatation, $\mathrm{OR}=1.8,95 \% \mathrm{Cl}$ : 1.56-2.07, $\mathrm{OR}=1.43,95 \% \mathrm{Cl}: 1.05-1.96$, respectively. They more often developed complications, $\mathrm{OR}=1.63,95 \% \mathrm{Cl}$ : 1.03-2.58, and had a higher number of PTCA in future, $\mathrm{OR}=6.77,95 \% \mathrm{Cl}: 5.73-7.99$ (Figures 1, 2).

\section{Comparison between coronary artery disease group (CAD) and control group (CG)}

The control group vs. coronary group in one dimensional analysis is characterized by more frequent presence of chronic kidney disease, $\mathrm{OR}=2.83,95 \% \mathrm{Cl}$ : 2.1-3.81, chronic obstructive pulmonary disease $\mathrm{OR}=$ $2.3,95 \% \mathrm{Cl}: 1.67-3.18$, fibrillation of atriums $\mathrm{OR}=3.02$, $95 \% \mathrm{Cl}: 2.25-4.07$ as well as caudation $\mathrm{OR}=1.49$, 95\% Cl: 1.09-2.01. The multivariate logistic regression seems to confirm these results (Figure 3 ).

\section{Discussion}

Indications for coronary angioplasty have become increasingly less controversial over the past few years because of two factors. First, the FFR technique is more and more commonly used and validated on a large number of subjects, which allows for precise and unambiguous selection of patients who will benefit from coronary angioplasty. Second, the new generation of stents coated with antimitotic drugs (DES) is more effective in prevention of restenosis. Recent studies indicate that interventional 


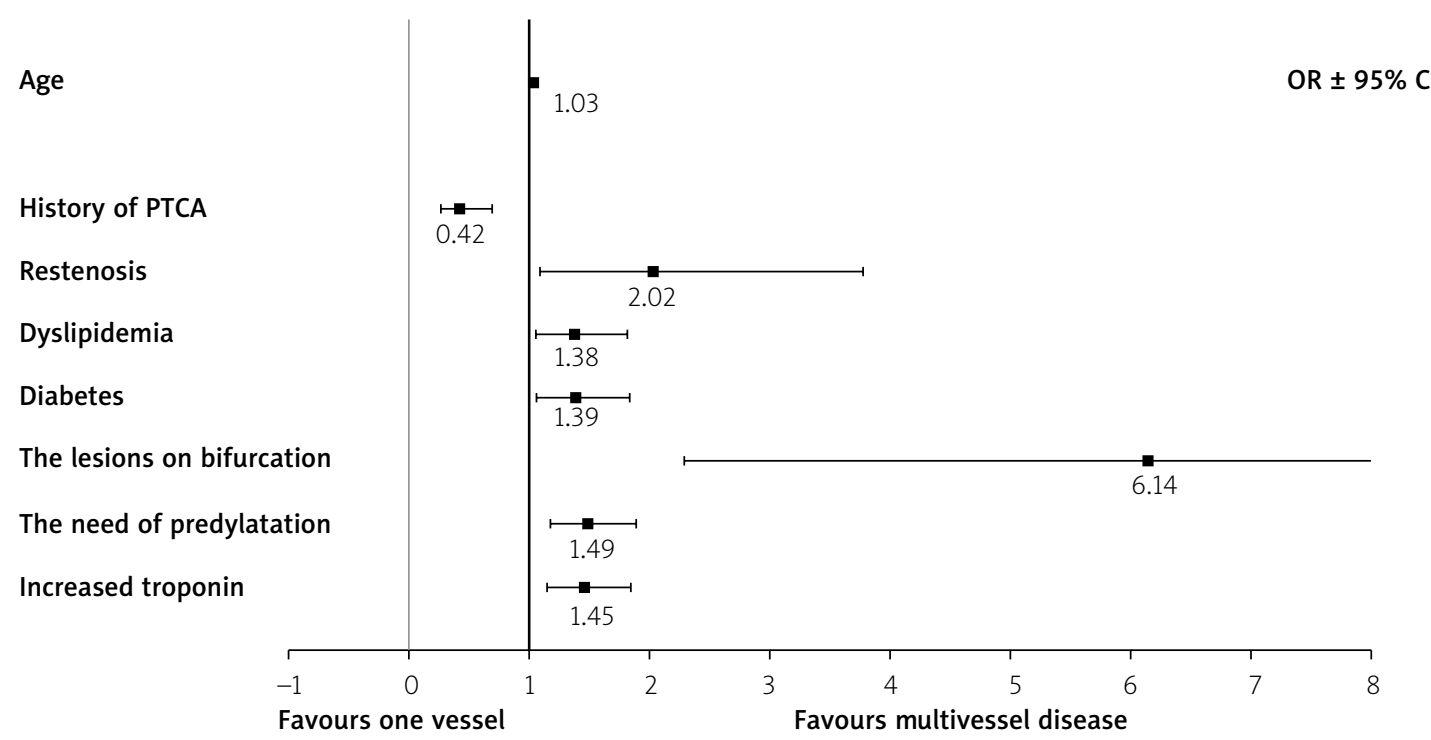

Figure 1. Comparison of subjects with significant stenosis in one coronary artery vs. multi-vessel disease during index angioplasty (multifactorial regression)

treatment of CAD combined with optimal pharmacotherapy is currently superior to pharmacotherapy alone [3, 8, 9].

Some patients with diagnosed CAD (with previous history of PTCA), despite the use of optimal medical therapy (OMT), experience reoccurring problems and need additional interventions. They are often qualified for second coronary angiography, because of the nature of the complaints and the results of initial diagnostic tests. Sometimes the patients require subsequent angioplasty, which is recommended in management of stable coronary syndromes by ESC guidelines $[3,5,6]$. This problem is not only interesting from a scientific point of view, but is also important because of its clinical, social and financial aspects.

Probably the main reason for these subsequent interventions is the natural progression of atherosclerosis $[3,5,6]$. The fact that the extent of atherosclerosis (multivessel disease) observed during index hospitalization was one of the strongest risk factors for future PTCA in our patients $(O R=12.1)$ supports that theory. Additionally, subjects with multivessel disease (found during index angioplasty), which is logical, needed predilatation and presented a 'bifurcation lesion' more frequently (OR $=6.14, \mathrm{OR}=1.49$ respectively). Given the complexity of the mechanisms responsible for the etiopathogenesis of restenosis, this phenomenon observed during index hospitalization in our patients was another strong risk factor for future coronary $(O R=2.02)$ interventions, and should also be interpreted as an exponent of an active and progressive atherosclerotic process.

Acceleration of atherosclerosis can be caused by non-compliance or non-adherence to OMT recommended in both primary and secondary prevention [5-7, 10]. The above is confirmed by the results of our study. Pa-

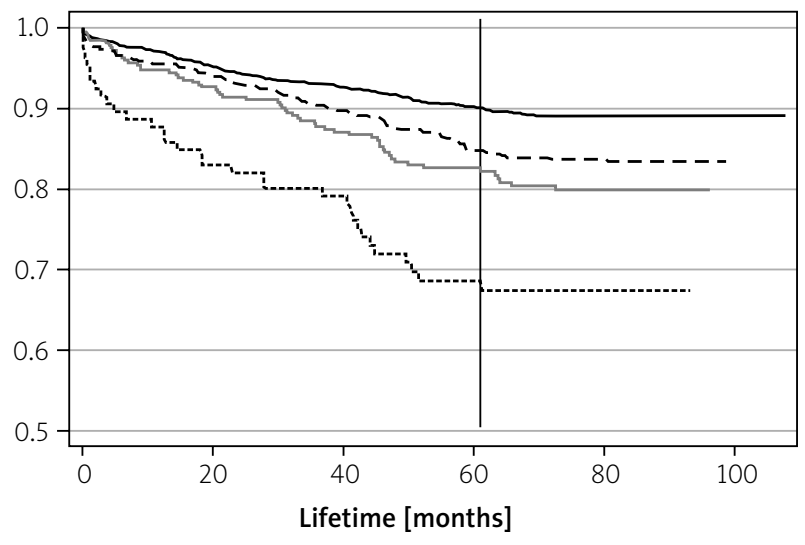

60 months survival in group:

— "One vessel" disease 91\% _ _Two vessels" disease 83\%

- - . "Three vessels" disease $87 \%$

----- "Three vessels" and co-branches 69\%

Figure 2. Survival curves depending on the number of critically narrowed vessels (one-vessel disease as a reference)

tients during index hospitalization as well as those who had subsequent interventions were characterized by very high LDL values, which were far above the therapeutic threshold recommended at that time by guidelines $(<70 \mathrm{mg} / \mathrm{dl}$ ). Despite the fact that $94 \%$ of patients had statins prescribed, the lipid-lowering therapy was inefficient.

The applied technique or type of implanted stents may also be conducive to qualification for subsequent invasive procedures [11]. However, our observation showed no association of either the procedural techniques (direct stenting or post dilatation) or the stents used (DES vs. BMS) with the need to perform coronary angioplasty in the future. It is interesting when we compare these re- 


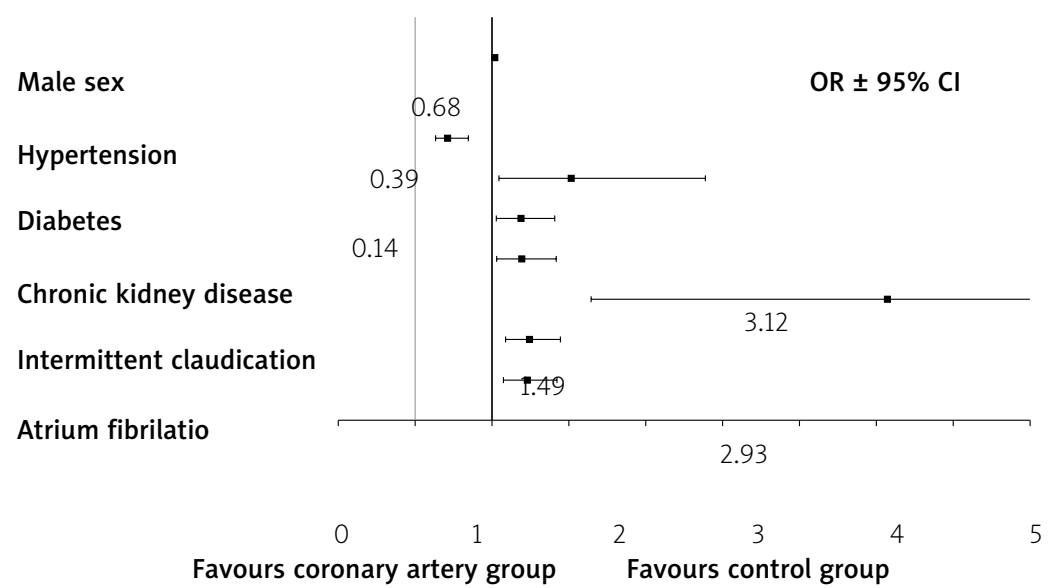

Figure 3. Multivariate regression logistic regression results for CAD vs. CG (reference group - CAD)

sults with findings of other Polish registries. DES was superior to BMS in impact on 1-year mortality in the STEMI group [12], but on the other hand the implantation of 2 DES was the cause of higher mortality and restenosis as opposed to implantation of 2 BMS [13].

Only the predilatation during index angioplasty was a significant factor for subsequent PTCA. The explanation for that phenomenon is probably the multiple inflation of the balloon catheter, preceding the implantation of a stent of the wrong length (stent lesion mismatch), which may result in the occurrence of stenosis in the proximal or distal part of the stent (candle effect).

On the other hand, we found that among all epicardial coronary arteries, significant stenosis in the left descending anterior was burdened with the strongest risk for subsequent PTCA, which is also confirmed by literature data [14-17]. Additionally, the more epicardial arteries were diseased, the greater was the mortality, which is also consistent with the literature. No such relationship was found in the number of performed angioplasties (data not provided).

An important parameter that can be used as a criterion to qualify someone for re-angiography/angioplasty is persistent ischemia which does not diminish despite the successful revascularization in the past, which in turn depends on the scope of the procedure (total or partial revascularization), changes in coronary microcirculation resistance, restenosis and other unknown factors [18-21]. In our study, indeed, restenosis was a risk factor predisposing to subsequent interventions $(\mathrm{OR}=$ 2.02-4.34).

Thirty percent of patients after successful intervention can still manifest symptoms of angina, which often promotes subsequent qualifications for coronary angiography [21-23]. This phenomenon could be a result of not sufficiently frequently performed assessment of partial coronary flow reserve - FFR, iFR - for other lesions or lesions in other arteries than those assessed.

There are also reports of coronary angiography in many people being caused by too hasty qualification of patients for this diagnostic procedure. This is not beneficial due to the social costs of these studies, especially in elderly subjects $[24,25]$. We found that some of the patients' complaints that became the basis for qualification for coronary angiography could have been associated with symptoms of chronic obstructive pulmonary disease, atrial fibrillation, intermittent claudication (for non-coronary cause), or chronic kidney disease. We found that these diseases were more common in the control group (without significant lesions in arteries) than in the group undergoing angioplasty during index hospitalization. It should be noted that potentially healthy control patients in our study differ from the average general population due to the presence of stenocardial complaints. In turn, the smaller amount of these burdens in subjects undergoing PTCA in our study raises hopes for a better prognosis for these patients, since diabetes, heart failure, chronic kidney disease and anemia are recognized as CAD progression factors [21].

Limitations of the study:

First, the study was a single-center retrospective analysis.

The project excluded patients with severe heart failure (NYHA III/IV), with left main stenosis, patients with extensive atherosclerotic lesions qualified for CABG, with concomitant heart defects and primary myocardial diseases.

The adopted criteria of lumen narrowing $>70 \%$ in diameter meant that the observation did not take into account the fate (observation of survival) of patients with atherosclerotic lesions in the range of 30 to $70 \%$, not requiring stenting according to current standards.

The population included patients with both ACS and stable coronary artery disease, and therefore was not homogeneous regarding anti-platelet therapy.

\section{Conclusions}

We found that the strongest risk factors for subsequent invasive procedures in patients were: critical ste- 
nosis of LAD at baseline coronary angiography, number of critically narrowed arteries (atherosclerotic burden), and restenosis in a previously implanted stents.

In the group of patients with CAD, a strong, positive relationship between total mortality and the number of critically altered coronary arteries (atherosclerotic burden) was observed. No similar association was found for the number of procedures.

Patients with symptoms suggestive of angina but with unobstructed arteries in subsequent coronary angiographies differed from CAD patients due to higher creatinine levels, more frequent chronic obstructive pulmonary disease, and more frequent symptoms of intermittent claudication.

\section{Acknowledgments}

Jaroslaw Hiczkiewicz and Paweł Burchardt - equal contribution to the manuscript.

Special thanks to Michal Michalak for expert statistical analysis and Simon Nowak for language service.

The study was support by statutory funds of the Faculty of Medicine and Health Sciences, University of Zielona Gora.

\section{Conflict of interest}

The authors declare no conflict of interest.

\section{References}

1. GBD 2013 Mortality and Causes of Death Collaborators. Global, regional, and national age-sex specific all-cause and cause-specific mortality for 240 causes of death, 1990-2013: a systematic analysis for the Global Burden of Disease Study 2013. Lancet 2015; 385: 117-71.

2. Statistics Poland, Demographic Survey Department. Life expectancy tables of Poland 2017. stat.gov.pl pp5. ISSN 1507-1340.

3. Knuuti J, Wijns W, Saraste A, et al.; ESC Scientific Document Group. 2019 ESC Guidelines for the diagnosis and management of chronic coronary syndromes: The Task Force for the diagnosis and management of chronic coronary syndromes of the European Society of Cardiology (ESC). Eur Heart J 2020; 41: 407-7.

4. Dudek D, Siudak Z, Grygier M, et al. Interventional cardiology procedures in Poland in 2018. Summary report of the Association of Cardiovascular Interventions of the Polish Cardiac Society (AISN PTK) and Jagiellonian University Medical College. Adv Interv Cardiol 2019; 15: 391-3.

5. Maddox TM, Stanislawski MA, Grunwald GK, et al. Nonobstructive coronary artery disease and risk of myocardial infarction. JAMA 2014; 312: 1754-63.

6. Varenhorst C, Hasvold P, Johansson S, et al. Culprit and nonculprit recurrent ischemic events in patients with myocardial infarction: data from SWEDEHEART (Swedish Web System for Enhancement and Development of Evidence-Based Care in Heart Disease Evaluated According to Recommended Therapies). J Am Heart Assoc 2018; 7: e007174.

7. Iderman EL, Kip KE, Whitlow PL, et al. Native coronary disease progression exceeds failed revascularization as cause of angina after five years in the bypass angioplasty revascularization investigation (BARI). J Am Coll Cardiol 2004; 44: 766-74.
8. Fearon WF, Nishi T, De Bruyne B, et al.; FAME 2 Trial Investigators. Fractional flow reserve-guided $\mathrm{PCl}$ versus medical therapy in stable coronary disease. N Engl J Med 2012; 367: 991-1001.

9. Ciccarelli G, Barbato E, Toth GG, et al. Angiography versus hemodynamics to predict the natural history of coronary stenoses: fractional flow reserve versus angiography in multivessel evaluation 2 substudy. Circulation 2018; 137: 1475-85.

10. Stone GW, Hochman JS, Williams DO, et al. Medical therapy with versus without revascularization in stable patients with moderate and severe ischemia. J Am Coll Cardiol 2016; 67: 81-99.

11. Mohan S, Dhall A. A comparative study of restenosis rates in bare metal and drug-eluting stents. Int I Angiol 2010; 19: e66-72.

12. Siudak Z, Dziewierz A, Rakowski T, et al. Borderline trend towards long-term mortality benefit from drug eluting stents implantation in ST-elevation myocardial infarction patients in Poland-data from NRDES registry. Catheter Cardiovasc Interv 2014; 83: 436-42.

13. Dziewierz A, Siudak Z, Rakowski T, et al. The impact of multiple stent implantation in the infarct-related artery on one-year clinical outcomes of patients with ST-elevation myocardial infarction undergoing primary percutaneous coronary intervention. Data from the Polish NRDES Registry. Kardiol Pol 2016; 74: 717-25.

14. Alfonso F, Cuesta J. The therapeutic dilemma of recurrent instent restenosis. Circ Cardiovasc Interv 2018; 11: e007109.

15. Alfonso F, Byrne RA, Rivero F, Kastrati A. Current treatment of instent restenosis. J Am Coll Cardiol 2014; 63: 2659-73.

16. Alfonso F, Pérez-Vizcayno MJ, Cárdenas A, et al.; RIBS IV Study Investigators. A prospective randomized trial of drug-eluting balloons versus everolimus-eluting stents in patients with in-stent restenosis of drug-eluting stents: the ribs iv randomized clinical trial. J Am Coll Cardiol 2015; 66: 23-33.

17. Baan J, Claessen BE, Dijk KB, et al. A randomized comparison of paclitaxel-eluting balloon versus everolimus-eluting stent for the treatment of any in-stent restenosis: the DARE Trial. JACC Cardiovasc Interv 2018; 11: 275-83.

18. Marinescu MA, Löffler Al, Ouellette M, et al. Coronary microvascular dysfunction, microvascular angina, and treatment strategies. JACC Cardiovasc Imaging 2015; 8: 210-20.

19. Camici PG, Crea F. Coronary microvascular dysfunction. N Engl J Med 2007; 356: 830-40.

20. Camici PG, d'Amati G, Rimoldi O. Coronary microvascular dysfunction: mechanisms and functional assessment. Nat Rev Cardiol 2015; 12: 48-62.

21. Landes $U$, Kornowski R, Assali A, et al. Predictors of long term outcomes in 11,441 consecutive patients following percutaneous coronary interventions. Am J Cardiol 2015; 115: 855-9.

22. Pocock SJ, Henderson RA, Seed P, et al. Quality of life, employment status, and anginal symptoms after coronary angioplasty or bypass surgery. 3-year follow-up in the Randomized Intervention Treatment of Angina (RITA) Trial. Circulation 1996; 94: 135-42.

23. Izzo P, Macchi A, De Gennaro L, et al. Recurrent angina after coronary angioplasty: mechanisms, diagnostic and therapeutic options. Eur Heart J Acute Cardiovasc Care 2012; 1: 158-69.

24. Patel MR, Peterson ED, Dai D, et al. Low diagnostic yield of elective coronary angiography. N Engl J Med 2010; 362: 886-95.

25. Chan PS, Patel MR, Klein LW, et al. Appropriateness of percutaneous coronary intervention. JAMA 2011; 306: 53-61. 